\title{
KRYSTYNA MIHUŁKA
}

Uniwersytet Rzeszowski

kmihulka@gmail.com

ORCID: 0000-0002-8665-4296

\author{
JOANNA CHOJNACKA-GÄRTNER \\ PWSZ w Koninie \\ chojnackagaertner@op.pl \\ ORCID: 0000-0002-3144-9965
}

\section{Forschungsbericht zum Profil eines "guten“ Fremdsprachenlehrers an der Hochschule}

\section{Profile of a 'good' foreign language teacher in a high school: Research report}

\begin{abstract}
The aim of this article is to present the profile of a 'good' foreign language teacher, which has been created on the basis of the comments made by about 230 students of modern languages at Polish institutions of higher education (colleges and universities) in two voivodeships of Poland, namely Podkarpackie Voivodeship and Wielkopolska Voivodeship. In this image, teacher personality traits, the majority of which are regarded as values not only in professional life (e.g. in the teaching profession), but also in personal life, have ranked the highest. Personality traits have overshadowed the other two groups of qualities, which foreign language teachers are also equipped with, i.e. didactic and glottodidactic ones. This study is part of a larger research project covering all types of schools in Poland (from primary schools, through middle and secondary schools, to colleges and universities).
\end{abstract}

KEYWORDS: teacher education, personality traits, didactic characteristics, glottodidactic characteristics.

SCHLÜSSELWORTE: Lehrerbildung, Persönlichkeitsmerkmale, didaktische Eigenschaften, glottodidaktische Eigenschaften. 


\section{EINLEITENDE BEMERKUNGEN}

Die vertieften Untersuchungen, die zum Ziel haben, das Bild eines idealen, herausragenden Lehrers (abgesehen vom unterrichteten Fach) zu skizzieren, gehen in Polen ${ }^{1}$ auf den Anfang des letzten Jahrhunderts zurück. In diesem Kontext ist der bahnbrechende wissenschaftliche Beitrag von Dawid (1912/1959) aus dem Jahr 1912 zu erwähnen, dessen Erwägungen sich grundsätzlich auf die Persönlichkeit des Lehrers stützten, die er als Seele der Lehrerschaft bezeichnete. Seine Ideen fanden ihre Fortsetzung u. a. in den Werken von Mysłakowski (1925/1959), Schuman (1947/1959), Kreuz (1947/1959), Baley (1958/1959) und Okoń (1959). Außer dem eher theoretischen Diskurs über einen guten Lehrer verdienen eine besondere Hervorhebung die ersten empirischen Untersuchungen, deren Autorinnen anhand der Ergebnisse der unter den Lehrern durchgeführten Umfrage (Dzierzbicka 1926) und der Beobachtung der berufstätigen Lehrer des Nachkriegspolens (Grzegorzewska 1947/2002) die notwendigen Eigenschaften eines guten Lehrers aufzählten und charakterisierten. Von der Annahme ausgehend, dass jeder gute Lehrer eine entsprechende Ausbildung besitzen sollte, konzentrierten sich die beiden Forscherinnen grundsätzlich auf die Charaktereigenschaften des Lehrers, die den Verlauf des didaktischen Prozesses determinieren und über seine Effekte entscheiden.

In der einschlägigen Literatur gibt es keine universelle Definition eines guten Lehrers. Die bis jetzt formulierten Auffassungen sind durch Dynamik gekennzeichnet, was verursacht, dass je nach der Entstehungszeit der Definition, andere Merkmale akzentuiert, andere dagegen in den Hintergrund gerückt werden. In den wissenschaftlichen Beiträgen, die bis zu den 60er Jahren des 20. Jahrhunderts veröffentlicht wurden, galt als ein guter, idealer Lehrer jemand, der über gewisse innerliche Werte verfügte, wie z. B.: Liebe der menschlichen Seelen (Dawid 1912/1959), das pädagogische Talent, dessen Grundlage die Kontaktbereitschaft und Kontaktfähigkeit bildeten (Mysłakowski 1925/1959), der Persönlichkeitsreichtum, dessen Form einerseits den Menschen von der Natur gegeben wird und andererseits von der Mühe, abhängt, das "Geschenkte" weiterzuentwickeln und zu bereichern (Szuman 1947/1959). In den nachfolgenden Beiträgen (z. B. Grzegorzewska 1947/2002; Kreuz 1947/1959) wurde ein guter, hervorragender Lehrer mit einem gut ausgebildeten und

\footnotetext{
${ }^{1} \mathrm{Im}$ vorliegenden Beitrag werden wegen der vorgegebenen begrenzten Textgröße die theoretischen Erwägungen nur dem polnischen Schulkontext gewidmet. Um diesen Blickwinkel um die ausländische, vorwiegend deutsche, Perspektive zu erweitern wird vorgeschlagen u. a. nach folgenden Veröffentlichungen zu greifen: Ausubel, Novak und Hanesian (1980), Düwell (1983), Kuen und Schwarzmann (2013), Hattie (2014), Kindler (2016), Loebell und Martzog (2016), Hattie und Zierer (2018a; 2018b).
} 
zugleich über eine breite Palette positiver Persönlichkeitseigenschaften (wie: Freundlichkeit, Güte, Sorge für jeden Schüler sowie das Gefühl der Verbundenheit mit ihm) verfügenden Menschen assoziiert. In den Veröffentlichungen aus den 50er Jahren des letzten Jahrhunderts wurde das Bild eines guten, perfekten Lehrers in folgende Komponente: Wissen, Fähigkeiten und Kompetenzen sowie Persönlichkeitsreichtum zerlegt (Okoń 1959: 14-15).

Die Persönlichkeit des Lehrers war im Zentrum des Interesses der polnischen Psychologen und Pädagogen bis zu den 60er Jahren des 20. Jahrhunderts. ${ }^{2}$ Seit diesem Zeitpunkt hält sich die Strömung, die Kompetenzen eines Lehrers zu erforschen, die über seine Eignung zum Lehrerberuf und seine Professionalität entscheiden. In den Beiträgen und wissenschaftlichen Monographien aus den 70er Jahren des letzten Jahrhunderts wurde jedoch neben der Fachqualifikationen (gemeint sind umfangreiches Weltwissen und die Bereitschaft zur systematischen Fortbildung), der didaktischen Geschicklichkeit (die auf die modellhafte Unterrichtsplanung und -durchführung, die Anwendung verschiedener Lernmaterialien und den Einsatz unterschiedlicher Lernmethoden zurückzuführen sind), der Gelehrsamkeit, noch die Persönlichkeit eines guten Lehrers als eines der Elemente genannt, die den Lernerfolg beeinflussen (Bandura 1972: 195). Seit den 80er Jahren des 20. Jahrhunderts überwiegt in der einschlägigen Literatur das Modell eines guten Lehrers, dass sich vorwiegend auf Fachqualifikationen und didaktische Kompetenzen beschränkt. Diese auch in der polnischen Glottodidaktik herrschende Tendenz findet ihren Ausdruck in vielen wissenschaftlichen Monographien ${ }^{3}$, Sammelbänden ${ }^{4}$ und Beiträgen, die dem Fremdsprachenlehrer gewidmet sind. Ihre Autoren stellen den Einfluss der Persönlichkeit des Lehrers auf die LehrerSchüler-Beziehung und damit auf den Verlauf des glottodidaktischen Prozesses zwar nicht in Frage, schenken ihm aber keine besondere Beachtung im Prozess der Wissensvermittlung, als ob die Effektivität des Unterrichtsgeschehens nur vom Wissen des (Fremdsprachen)Lehrers abhinge. Diese Perspektive wurde auch von den Autoren vertreten, die den Rahmen der Bildungsbedingungen angehender Fremdsprachenlehrer in Polen festlegten.

In Polen wurden 2012 mit der Verordnung des Ministers für Wissenschaft und Hochschulwesen (MNiSW) die Standards für die Lehrerbildung in Bezug auf alle zu unterrichtenden Fächer, unter anderem auch Fremdsprachen, ein-

\footnotetext{
2 Über den Einfluss der Persönlichkeit des Lehrers auf den Bildungsprozess kann man u. a. in Mihułka (2018a; 2018b) lesen.

${ }^{3}$ Siehe z. B. Grucza, Krumm \& Grucza (1993), Pfeiffer (2001), Wysocka (2003), Zawadzka (2004).

${ }^{4}$ Siehe u. a. Grucza (1988), Aleksandrowicz-Pędich \& Komorowska (1999), Pawlak, Mystkowska-Wiertelak \& Pietrzykowska (2009), Stańczyk (2013), Andrzejewska (2015a; 2015b), Wawrzyniak-Śliwska (2013).
} 
geführt. Ihre Gültigkeit läuft Ende September 2018 ab. Das Dokument bildet die gesetzlichen Grundlagen für die Ausbildung der Lehrkräfte in Polen und greift die Problematik im Hinblick auf folgende Disziplinen auf: Psychologie, Pädagogik, Allgemeine Didaktik, Fachdidaktik sowie Praktika. Darüber hinaus präzisieren die Standards die sogenannten Ausbildungsergebnisse, die die Absolventen nach Abschluss des Studiums mit Spezialisierung auf Lehramt erzielen sollen. Die werden in dem Dokument in Form von zwei separaten Katalogen als allgemeine und detaillierte Ergebnisse präsentiert, wobei die zweite Gruppe weiter in drei Kategorien, das heißt Wissen, Fertigkeiten und Sozialkompetenz unterteilt wird. In beiden Listen werden Kompetenzen und Fähigkeiten genannt, die einen Lehrer bzw. Fremdsprachenlehrer auszeichnen sollen. Sie beziehen sich aber grundsätzlich auf sein didaktisches Handeln im Unterrichtsgeschehen. Es kommt ihnen „eine Orientierungsfunktion in Bezug auf die Kompetenzen $\mathrm{zu}$, die im Laufe der Ausbildung zu erwerben sind, sowie auf die Organisation der Studiengänge und die zu behandelnden Inhalte“ (Krzysiak 2013: 161). Die genauen Themenbereiche werden im weiteren Teil des Dokuments angesprochen, das fünf Module (obligatorische und fakultative) bilden. Es handelt sich dabei um folgende Module:

- Modul 1: Fachwissenschaft,

- Modul 2: Psychologie und Pädagogik,

- Modul 3: Allgemeine Didaktik und Fachdidaktik,

- Modul 4: 2. Fachwissenschaft und ihre Fachdidaktik,

- Modul 5: Sonderpädagogik (MNiSW 2012: 1-37; Krzysiak 2016: 136-139; Krzysiak 2013: 156-162).

In jedem der oben genannten Module wird genau bestimmt, wie viele Stunden man für einen bestimmten im Studium für die Lehrerbildung vorgesehenen Bereich braucht, um die erforderlichen Ausbildungsergebnisse zu erreichen sowie wie viele ECTS-Punkte jedem Modul zugeschrieben werden sollten. Der kurzen Beschreibung jedes einzelnen Moduls folgen dann die Bildungsinhalte, die schon ein sehr detailliertes Spektrum von den im Studium zu behandelnden Themen enthalten (MNiSW 2012: 1-37; Krzysiak 2016: 136-139; Krzysiak 2013: 156-162).

Obwohl jedoch die oben beschriebenen Standards alle Themenbereiche ansprechen, mit denen die werdende Lehrkraft in ihrem Studium in allen in Modulen der Lehrerbildung erfassten Fächern konfrontiert werden sollte, um auf ihre spätere Arbeit gut vorbereitet zu werden, offenbaren sie nicht, welche Personen sich als besonders geeignet für den Lehrerberuf erweisen sowie welche Persönlichkeitsmerkmale bei der Ausübung dieser Tätigkeit als unentbehrlich fungieren. ${ }^{5}$

${ }^{5}$ Das vom Ministerium verfasste Dokument enthält noch weitere Schwächen, die von Krzysiak (2016: 139) erwähnt wurden, und zwar, die oben angedeuteten allgemeinen und 
In Bezug auf die obigen Ausführungen verfolgt der vorliegende Beitrag das Ziel, das Profil eines "guten“ Fremdsprachenlehrers zur Diskussion zu stellen. Die Autorinnen versuchten im Schul-/Studienjahr 2016/2017 auf jeder Bildungsstufe in Polen 6 , von der Grundschule an, über das Gymnasium und die Oberschule bis zur Hochschule ${ }^{7}$, die Informationen zu sammeln, welche Merkmale ein Fremdsprachenlehrer besitzen soll, um von seinen Schülern als "gut" bezeichnet zu werden. Insgesamt waren es 841 Personen, die eine in ihrer Muttersprache schriftlich gestellte offene Frage Jaki powinien być "dobry" nauczyciel jezzyka obcego? Jakie powinien posiadać cechy? [,Wie soll der "gute" Fremdsprachenlehrer sein? Welche Eigenschaften soll er besitzen?'] stichwortartig oder in Form von einer längeren Beschreibung auf Polnisch beantworteten. ${ }^{8}$

An dieser Stelle soll man hinzufügen, dass an der in diesem Forschungsbericht beschriebenen Untersuchung ausschließlich die Hochschullerner teilnahmen, die sich erstens für eine neuphilologische Studienrichtung entschieden und zweitens selber nach ihrem Studium in den Lehrerberuf eintreten wollen. Deswegen war ihre Beteiligung an dem Projekt für beide Forscherinnen von immenser Bedeutung. Für die Datenerhebung wurden zwei gegenüberliegende Regionen Polens gewählt: das Karpatenvorland, das von den Studenten der Universität Rzeszów und der PWSZ in Krosno repräsentiert wurde sowie Großpolen, das die Studenten der PWSZ in Konin vertraten. Die Probanden, denen die Anonymität der Antworten gesichert wurde, nahmen an der Untersuchung freiwillig teil. Die Befragung wurde nur ein-

detaillierten Ausbildungsergebnisse sind, ihrer Meinung nach, nicht kohärent und wenig ausgereift und dies kann den Nutzern von den einzelnen Listen Schwierigkeiten bereiten. Außerdem erweisen sich die beschriebenen Kompetenzen als schwer messbar, „es sei denn, dass die Anwender (...) selbst die komplexen Kompetenzen auffächern und das zu erwartende Leistungsniveau festlegen“ (Krzysiak 2016: 139), was, laut der Autorin, zu Interpretationsproblemen der einzelnen Kompetenzbeschreibungen führen und „eine einheitliche Umsetzung der Lehrerbildungsstandards in Polen problematisch machen kann“ (Krzysiak 2016: 139).

${ }^{6}$ Die in den einzelnen Schultypen gewonnenen Daten (841 Probanden) wurden in Form von vier (abgesehen von dem vorliegenden) unterschiedlichen Forschungsberichten in Druck gegeben, die wie folgt betitelt sind: Forschungsbericht zum Profil eines "guten“ Fremdsprachenlehrers in der Grundschule (Mihułka \& Chojnacka-Gärtner 2017b); Forschungsbericht zum Profil eines "guten“ Fremdsprachenlehrers am Gymnasium (Mihułka \& Chojnacka-Gärtner 2017a); Forschungsbericht zum Profil eines "guten" Fremdsprachenlehrers in der Oberschule (Mihułka \& Chojnacka-Gärtner 2018); „Auf die Persönlichkeit des Lehrers kommt alles an [...]" - Forschungsbericht zum Profil eines "guten“ Fremdsprachenlehrers in allen Schultypen in Polen (Mihułka \& Chojnacka-Gärtner 2019).

7 An der Untersuchung nahmen die Probanden im Alter von 10 bis 25 Jahren teil.

8 Wegen der großen Altersdifferenzen der untersuchten Personen und ihrer davon abhängenden kognitiven sowie emotionalen Entwicklung, wurde die Forschungsfrage sehr allgemein formuliert, um allen Beteiligten das Verständnis des Gemeinten zu vereinfachen (d. h. ohne Berücksichtigung der Persönlichkeitsmerkmale und ohne Formulierung der fachwissenschaftlichen Kompetenzen eines "guten” Lehrers). 
mal durchgeführt ${ }^{9}$, wobei anzumerken wäre, dass Untersuchungen dieser Art, mit nur einem Messzeitpunkt in der einschlägigen Literatur als Querschnittdiagnose (Komorowska 1982: 172-190), Querschnitterhebung (Diekmann 2007: 194) oder als eine statische Untersuchung (Wilczyńska \& Michońska-Stadnik 2010: 62-63) bezeichnet werden.

Darüber hinaus gilt die zusammengestellte Stichprobe als eine Zielstichprobe, weil die gewählten Probanden bestimmte für die Untersuchung relevante Kriterien erfüllen mussten. Das waren, unter anderem:

- neuphilologische Studienrichtung, Spezialisierung auf Lehramt,

- Lage der Hochschule (zwei gegenüberliegende Regionen Polens),

- Alter der Lernenden (19-25 Jahre),

- Kontakt mit zumindest zwei Fremdsprachen während der Bildung.

Dank einer der offenen Techniken der Datenerhebung, d. h. der Methode der freien Assoziation, wurden bedeutsame Daten gewonnen, die sowohl eine qualitative als auch quantitative Analyse des gesammelten Untersuchungsmaterials ermöglichten.

\section{ZUR DATENERHEBUNG UND -AUSWERTUNG}

Wie schon oben angedeutet, beteiligten sich an der Untersuchung insgesamt 841 Personen, wobei sich die Gruppe der Hochschullerner, mit denen sich dieser Beitrag beschäftigt, aus 229 Probanden zusammensetzte: 129 Philologie-Studenten aus dem Karpatenvorland und 100 aus Großpolen.

Die Lernenden nannten zu der ihnen schriftlich gestellten Frage spontan eingefallene Assoziationen, die unterschiedliche Formen annahmen. Die Mehrheit von ihnen wählte als Antwort einfache Adjektive zur Persönlichkeitsbeschreibung (Aufzählung der Merkmale), einige beantworteten die Frage in Form von einer in Sätzen verfassten Äußerung und ein paar Studenten schrieben sogar kurze Aufsätze zu dem angegebenen Thema. Da die Studenten verschiedene neuphilologische Studienrichtungen repräsentierten, wurden sie gebeten, ihre Antworten in der Muttersprache aufzuschreiben, die für die Zwecke dieses Beitrags von beiden Forscherinnen ins Deutsche übersetzt wurden.

\subsection{Quantitative Datenanalyse}

Die von den Studierenden genannten Merkmale eines "guten“ Lehrers sowie die prozentual berechnete Häufigkeit des Auftretens der einzelnen

\footnotetext{
${ }^{9}$ Die Untersuchung fand im April 2017 statt.
} 
Eigenschaften wurden in der Tabelle 1 dargestellt, wobei in der zweiten Spalte die im Karpatenvorland gewonnenen Daten (Gruppe I) und in der dritten die aus Großpolen (Gruppe II) präsentiert wurden..$^{10}$ Die letzte Spalte enthält die Zusammenstellung der Daten aus beiden untersuchten Regionen Polens. An dieser Stelle muss man noch erwähnen, dass bei der quantitativen Analyse des untersuchten Materials ausschließlich die Antworten berücksichtigt wurden, die in der Gesamtauswertung von mindestens 5 Prozent der Befragten gegeben wurden. Es wäre noch anzumerken, dass beim Beantworten der Untersuchungsfrage sich die Probanden mehr auf die Ausdruckskraft der Äußerung als auf die Bedeutungspräzision konzentrierten, deswegen erhalten ihre Arbeiten viele allgemeine, mehrdeutige Wörter, die sich durch große phraseologische Konnektivität und emotionale Färbung auszeichnen.

Tabelle 1. Das Bild eines „guten“ Fremdsprachenlehrers aus der Perspektive der Hochschullerner

\begin{tabular}{|l|c|c|c|}
\hline \multicolumn{4}{|c|}{ Der Fremdsprachenlehrer soll: } \\
\hline \multicolumn{1}{|c|}{ Eigenschaft / Merkmal } & $\begin{array}{c}\text { Gruppe I } \\
\text { in \% }\end{array}$ & $\begin{array}{c}\text { Gruppe II } \\
\text { in \% }\end{array}$ & $\begin{array}{c}\text { Gesamtanteil } \\
\text { in \% }\end{array}$ \\
\hline geduldig sein & 42,6 & 52 & 46,7 \\
\hline verständnisvoll sein & 44,9 & 46 & 45,4 \\
\hline gerecht sein & 24 & 43 & 32,3 \\
\hline ideenvoll / kreativ sein & 32,6 & 32 & 32,3 \\
\hline gut erklären / das Wissen geschickt vermitteln & 40,3 & 10 & 27 \\
\hline anspruchsvoll / streng sein & 26,4 & 11 & 19,6 \\
\hline zum Fremdsprachenlernen motivieren / anspornen & 25,6 & 4 & 16,2 \\
\hline seinen Beruf gern ausüben / Lehrer aus Leidenschaft sein & 28 & 7 & 15,3 \\
\hline hilfsbereit sein & 10,8 & 21 & 15,3 \\
\hline Sinn für Humor haben / fröhlich, lustig sein & 11,6 & 19 & 14,8 \\
\hline kompetent sein & 10,1 & 20 & 14,4 \\
\hline $\begin{array}{l}\text { das Interesse an dem Fach bei seinen Schülern wecken } \\
\text { können }\end{array}$ & 22,5 & 3 & 13,9 \\
\hline den Unterricht interessant durchführen & 20,9 & 3 & 13,5 \\
\hline nett / sympathisch sein & 8,5 & 19 & 13,1 \\
\hline
\end{tabular}

${ }^{10}$ Die in den einzelnen Regionen Polens erreichten Ergebnisse wurden im vorliegenden Beitrag wegen der begrenzten Textgröße nicht erläutert und miteinander verglichen. In der Tabelle wurde jedoch die regionale Einteilung erhalten, um auf teilweise große Schwankungen in den Antworten der Befragten von beiden Gruppen hinzuweisen. 


\begin{tabular}{|c|c|c|c|}
\hline \multicolumn{4}{|c|}{ Der Fremdsprachenlehrer soll: } \\
\hline Eigenschaft / Merkmal & $\begin{array}{l}\text { Gruppe I } \\
\text { in } \%\end{array}$ & $\begin{array}{c}\text { Gruppe II } \\
\text { in } \%\end{array}$ & $\begin{array}{c}\text { Gesamtanteil } \\
\text { in } \%\end{array}$ \\
\hline $\begin{array}{l}\text { verschiedene Methoden des Unterrichtens einsetzen / } \\
\text { einen abwechslungsreichen Unterricht durchführen }\end{array}$ & 20,9 & 0 & 11,8 \\
\hline objektiv / unparteiisch sein & 6,2 & 18 & 11,3 \\
\hline positiv / freundschaftlich zu den Schülern eingestellt sein & 14,7 & 7 & 11,3 \\
\hline $\begin{array}{l}\text { das Wissen über die Kultur des Landes besitzen, dessen } \\
\text { Sprache er unterrichtet }\end{array}$ & 18,6 & 0 & 10,5 \\
\hline jeden Schüler individuell behandeln & 15,5 & 1 & 9,2 \\
\hline schüleroffen sein & 13,9 & 3 & 9,2 \\
\hline $\begin{array}{l}\text { nicht nur anhand des Lehrwerks unterrichten, sondern } \\
\text { zusätzliche didaktische Hilfsmaterialien in den Unterricht } \\
\text { einbeziehen }\end{array}$ & 16,3 & 0 & 9,2 \\
\hline gut / perfekt die Sprache beherrschen, die er unterrichtet & 12,4 & 4 & 8,7 \\
\hline $\begin{array}{l}\text { umfangreiches Wissen über die Sprache haben, die er } \\
\text { unterrichtet }\end{array}$ & 11,6 & 5 & 8,7 \\
\hline unterschiedliche Lehrmittel im Unterricht einsetzen & 14,7 & 1 & 8,7 \\
\hline einen guten Kontakt zu den Schülern haben & 13,9 & 1 & 8,2 \\
\hline kommunikativ sein & 6,9 & 10 & 8,2 \\
\hline einfühlsam / emphatisch sein & 3,1 & 15 & 8,2 \\
\hline diszipliniert / gut organisiert sein & 10,1 & 5 & 7,8 \\
\hline konsequent sein (bei der Bewertung; in seinem Handeln) & 6,2 & 10 & 7,8 \\
\hline motiviert sein, sich weiterzubilden & 7,7 & 6 & 6,9 \\
\hline ehrlich sein & 5,4 & 8 & 6,5 \\
\hline engagiert sein & 10,8 & 0 & 6,1 \\
\hline eine gute Aussprache haben (ohne Akzent sprechen) & 8,5 & 1 & 5,2 \\
\hline $\begin{array}{l}\text { die Schüler zum Sprechen motivieren / einen großen } \\
\text { Wert auf die Kommunikativität der Schüler legen }\end{array}$ & 10 & 0 & 5,6 \\
\hline $\begin{array}{l}\text { das Unterrichtstempo, -methoden und -aufgaben dem } \\
\text { Sprachniveau der Schüler anpassen }\end{array}$ & 10 & 0 & 5,6 \\
\hline im Unterricht Spiele einsetzen & 10,1 & 0 & 5,6 \\
\hline für Ruhe und Disziplin im Unterricht sorgen & 8,5 & 2 & 5,6 \\
\hline ruhig/ gelassen/ ausgeglichen sein & 6,9 & 3 & 5,2 \\
\hline
\end{tabular}

Aus der dargestellten Tabelle geht hervor, dass die Studierenden für einen "guten" Lehrer grundsätzlich eine Person halten, die in der Gesellschaft auch als ein "guter" Mensch gilt. Auf dem Siegerpodest befinden sich nämlich solche Eigenschaften wie geduldig (46,7 Prozent), verständnisvoll 
(45,4 Prozent) und auf dem dritten Platz ex aequo gerecht und ideenvoll / kreativ (32,3 Prozent). Erst an der vierten Stelle mit 27 Prozent der Antworten wird ein Merkmal platziert, das mit der Lehrerausbildung in Korrelation steht und zwar, der Fremdsprachenlehrer sollte das Wissen geschickt vermitteln und den Lernstoff verständlich und ruhig erklären. Darüber hinaus sollte er hilfsbereit (15,3 Prozent), trotzdem aber streng und anspruchsvoll sein, auch sich selbst gegenüber (19,6 Prozent).

Für das Unterrichtsgeschehen ist es von besonderer Bedeutung, mit einem Lehrer konfrontiert zu werden, der im Stande ist, seine Schüler zum Fremdsprachenlernen zu motivieren (16,2 Prozent). Und das kann laut 15,3 Prozent der Probanden nur ein Mensch erreichen, der Lehrer aus Leidenschaft ist, d. h. jemand, der seinen Beruf mag und gerne ausübt, jemand, der Sinn für Humor hat, immer fröhlich, nett / sympathisch und höflich (zwischen 13 und 14 Prozent) sowie objektiv / unparteiisch ist (11,3 Prozent). Ferner sollte sein Unterricht interessant, unter Einbeziehung von unterschiedlichen Unterrichtsmethoden, verschiedenen Hilfsmaterialien und gleichzeitig mit einer positiven, partnerschaftlichen Einstellung $\mathrm{zu}$ den Lernenden gestaltet werden (zwischen 9 und 11 Prozent). Für mehr als 9 Prozent der Untersuchungspersonen war die Schüleroffenheit des Lehrers relevant sowie die Tatsache, dass jeder Lernende von ihm wie ein Individuum behandelt werden sollte.

Die Zusammenstellung der für die Studierenden wichtigen Lehrermerkmale zeigt außerdem, dass für 10,5 Prozent der Befragten die Kulturkompetenz des Lehrers eine wichtige Rolle spielte. Seine sprachliche sowie seine metasprachliche Kompetenz schätzten lediglich 8,7 Prozent der Studenten. Die eingehenden Lehrer erwähnten im Weiteren solche Eigenschaften wie Kommunikativität, Empathie, Diszipliniertheit, Ehrlichkeit, Engagiertheit, Motiviertheit als Merkmale, die einen "guten" Fremdsprachenlehrer kennzeichnen sollten (zwischen 6 und 8 Prozent).

Am Ende der Tabelle 1 befinden sich weitere im Fremdsprachenlehrerberuf erwähnenswerte Merkmale, die von 5 Prozent der Untersuchungspersonen genannt wurden. Der Fremdsprachenlehrer sollte den Probanden zufolge eine gute Aussprache haben, bestenfalls ohne Akzent sprechen. Des Weiteren sollte er Fähigkeiten bzw. Eigenschaften besitzen, die direkt im Unterricht zum Vorschein kommen, wie z. B. die Fähigkeit, unter Einbeziehung von Spielen zu unterrichten, die Schüler zum Sprechen zu motivieren sowie Aufgaben, Arbeitstempo und Unterrichtsmethoden dem sprachlichen Niveau der Lernenden anzupassen. Im Unterricht sollte die Lehrkraft die Ruhe bewahren sowie die Disziplin herstellen und aufrechterhalten.

Die quantitative Analyse des gesammelten Datenmaterials (Tabelle 1) zeigt deutlich, dass in dem Profil eines "guten“ Fremdsprachenlehrers unter 
Studenten, zukünftigen Lehrern, die Persönlichkeitsmerkmale die Oberhand gewinnen. Unter den ersten zehn Merkmalen befinden sich nämlich sechs, die jeden beliebigen Menschen kennzeichnen könnten (geduldig, verständnisvoll, gerecht, ideenvoll, humorvoll und hilfsbereit). Diese Dominanz war schon auf den anderen untersuchten Bildungsstufen festzustellen (Grundschule, Gymnasium, Oberschule $\left.{ }^{11}\right)$, jedoch muss man unterstreichen, dass bei der in diesem Beitrag beschriebenen Studentengruppe viel häufiger die Fachqualifikationen und die didaktischen Kompetenzen genannt wurden, die für den Lehrerberuf, insbesondere den Fremdsprachenlehrerberuf, charakteristisch sind.

\subsection{Qualitative Datenanalyse}

Ein gründliches Bild eines „guten“ Fremdsprachenlehrers liefert die qualitative Analyse der erhobenen Daten, während derer sowohl die Merkmale, die den Gegenstand der quantitativen Analyse bildeten als auch diejenigen, die wegen ihrer zu niedrigen Auftretenshäufigkeit in der quantitativen Analyse unbeachtet blieben, einer Interpretation unterzogen wurden. Die von zumindest zwei Probanden zum Ausdruck gebrachten Eigenschaften schildert die Tabelle 2, wobei anzumerken wäre, das all die genannten Merkmale in drei Gruppen eingeteilt wurden. Die erste Spalte enthält die Persönlichkeitsmerkmale, die jeden beliebigen Menschen (abgesehen von seiner beruflichen Tätigkeit) beschreiben könnten, der bei anderen großes Ansehen genießt, respektiert wird und in der Gesellschaft generell als ein "guter" Mensch fungiert. In der zweiten Spalte werden die didaktischen Eigenschaften aufgezählt, die jeden "guten“ Lehrer, unabhängig von dem von ihm unterrichteten Fach, kennzeichnen sollten. Und in der dritten Spalte, werden glottodidaktische Merkmale aufgelistet, die ausschließlich zu dem Bild eines "guten" Fremdsprachenlehrers passen.

An dieser Stelle sollte man noch hervorheben, dass viele von den Studenten aufgeschriebene Merkmale zusätzlich mit einem Kommentar, einem Beispiel bzw. einer kurzen Erklärung des Gemeinten versehen wurden. Eben diese Aussagen der Probanden machen unserer Ansicht nach das anhand der quantitativen Datenanalyse erstellte (siehe Tabelle 1) und durch die Äußerungen einiger Befragten ergänzte (siehe Tabelle 2) Bild eines "guten" Fremdsprachenlehrers noch vollständiger, denn es wurde um emotionale und affektive Elemente bereichert.

${ }^{11}$ Siehe dazu Mihułka \& Chojnacka-Gärtner (2017a; 2017b; 2018). 
Tabelle 2. Merkmale eines "guten“ Fremdsprachenlehrers aus der Sicht der Hochschullerner

\begin{tabular}{|c|c|c|}
\hline \multicolumn{3}{|c|}{ Der "gute" Fremdsprachenlehrer } \\
\hline $\begin{array}{l}\text { Persönlichkeits- } \\
\text { eigenschaften }\end{array}$ & & Glottodidaktische Eigenschaften \\
\hline $\begin{array}{l}\text { - ist geduldig } \\
\text { - ist verständnisvoll } \\
\text { - ist gerecht } \\
\text { - ist kreativ / ideenvoll } \\
\text { - ist anspruchsvoll } \\
\text { - ist hilfsbereit } \\
\text { - hat Sinn für Humor } \\
\text { - ist nett / sympathisch } \\
\text { - ist objektiv / unparteiisch } \\
\text { - ist kommunikativ } \\
\text { - ist emphatisch / ein- } \\
\text { fühlsam } \\
\text { - ist diszipliniert / gut } \\
\text { organisiert } \\
\text { - ist konsequent } \\
\text { - ist motiviert / sich } \\
\text { weiterzubilden } \\
\text { - ist gründlich } \\
\text { - ist engagiert } \\
\text { - ist ruhig / gelassen / } \\
\text { ausgeglichen } \\
\text { - ist tolerant } \\
\text { - ist pünktlich } \\
\text { - mag Arbeit mit Men- } \\
\text { schen } \\
\text { - ist eloquent } \\
\text { - ist flexibel } \\
\text { - erweckt keine Furcht / } \\
\text { Angst } \\
\text { - ist ehrlich } \\
\text { - ist standhaft } \\
\text { - ist selbstbewusst } \\
\text { - ist intelligent } \\
\text { - ist durchsetzungsfähig } \\
\text { - ist fröhlich / heiter / } \\
\text { lächelnd } \\
\text { - nimmt sich nicht zu } \\
\text { ernst } \\
\text { - hat Prinzipien / an die } \\
\text { er sich hält } \\
\text { - ist perfektionistisch / } \\
\text { professionell } \\
\text { - ist locker } \\
\text { - ist fürsorglich } \\
\text { - ist zäh }\end{array}$ & $\begin{array}{l}\text { - erklärt gut / vermittelt geschickt das Wissen } \\
\text { - unterrichtet nicht nur anhand eines Lehr- } \\
\text { werks, sondern bezieht zusätzliche didakti- } \\
\text { sche Hilfsmaterialien in den Unterricht ein } \\
\text { - vermittelt den Schülern zusätzliche interes- } \\
\text { sante Informationen zum Unterrichtsthema } \\
\text { - teilt eigene Erfahrungen mit seinen Schülern } \\
\text { - weckt das Interesse an dem Fach bei seinen } \\
\text { Schülern } \\
\text { - führt den Unterricht interessant durch } \\
\text { - setzt verschiedene Methoden des Unter- } \\
\text { richtens ein } \\
\text { - setzt im Unterricht verschiedene Sozialfor- } \\
\text { men ein } \\
\text { - setzt im Unterricht Spiele ein } \\
\text { - führt im Unterricht abwechslungsreiche } \\
\text { Aufgaben durch } \\
\text { - überprüft oft das Wissen seiner Schüler } \\
\text { - ist konsequent (bei der Bewertung) } \\
\text { - berücksichtigt bei der Bewertung das En- } \\
\text { gagement des Schülers } \\
\text { - bewertet das Wissen seiner Schüler, nicht } \\
\text { ihre Persönlichkeit } \\
\text { - gibt den Lernenden die Chance, die Noten } \\
\text { zu verbessern } \\
\text { - ist auf den Unterricht immer gut vorbereitet } \\
\text { - ist kompetent } \\
\text { - kennt sich mit der neuesten Technologie / } \\
\text { mit den neuesten Medien aus } \\
\text { - verfügt über großes Wissen / ist belesen } \\
\text { - hat eine gute Ausbildung } \\
\text { - besitzt pädagogische Fähigkeiten } \\
\text { - ist Lehrer aus Leidenschaft / mag seine } \\
\text { Arbeit / mag das, was er tut } \\
\text { - sorgt für Ruhe und Disziplin im Unterricht } \\
\text { - sorgt für gute Atmosphäre im Unterricht } \\
\text { - ist positiv / freundschaftlich zu seinen } \\
\text { Schülern eingestellt / hat eine richtige Ein- } \\
\text { stellung den Schülern gegenüber } \\
\text { - behandelt jeden Schüler individuell } \\
\text { - ist schüleroffen } \\
\text { - hat einen guten Kontakt zu den Schülern } \\
\text { - hört den Schülern zu / ist offen für ihre } \\
\text { Ideen / Vorschläge } \\
\text { - mag Kinder / Schüler }\end{array}$ & $\begin{array}{l}\text { - beherrscht gut / perfekt die } \\
\text { Sprache, die er unterrichtet / } \\
\text { kann fließend in der Fremd- } \\
\text { sprache, die er unterrichtet, } \\
\text { kommunizieren } \\
\text { - hat eine gute Aussprache } \\
\text { (spricht ohne Akzent) } \\
\text { - verfügt über reiches Vokabular } \\
\text { - hat umfangreiches Wissen über } \\
\text { die Sprache, die er unterrichtet } \\
\text { - besitzt das Wissen über die } \\
\text { Kultur (Geschichte, Literatur, } \\
\text { Traditionen usw.) des Landes, } \\
\text { dessen Sprache er unterrichtet } \\
\text { - ist Experte in seinem Fach } \\
\text { - ist kompetent in Bezug auf die } \\
\text { Fremdsprachenvermittlung } \\
\text { - erklärt gut Grammatik, } \\
\text { - motiviert zum Fremdsprachen- } \\
\text { lernen } \\
\text { - motiviert die Schüler zum } \\
\text { Sprechen / legt einen großen } \\
\text { Wert auf die Kommunikativität } \\
\text { der Schüler } \\
\text { - achtet auf die praktische An- } \\
\text { wendung einer Fremdsprache } \\
\text { - benutzt im Unterricht aus- } \\
\text { schließlich die Fremdsprache, } \\
\text { - kann eigene sprachliche Fehler } \\
\text { zugeben } \\
\text { - korrigiert die sprachlichen } \\
\text { Fehler der Schüler bzw. lässt } \\
\text { die Selbstkorrektur zu und ach- } \\
\text { tet auf die Aussprache der } \\
\text { Schüler (positive Korrektur) } \\
\text { - passt das Unterrichtstempo } \\
\text { - methoden und aufgaben dem } \\
\text { Sprachniveau der Schüler an } \\
\text { - passt die Lernmethode bzw. die } \\
\text { sprachliche Übung dem Niveau } \\
\text { der Schüler an } \\
\text { - zeigt den Schülern die effek- } \\
\text { tivsten Methoden / Strategien } \\
\text { der Fremdsprachenaneignung } \\
\text { (z. B. im Wortschatzbereich) }\end{array}$ \\
\hline
\end{tabular}




\begin{tabular}{|l|l|l|}
\hline \multicolumn{2}{|c|}{ Der "gute“ Fremdsprachenlehrer } \\
\hline $\begin{array}{c}\text { Persönlichkeits- } \\
\text { eigenschaften }\end{array}$ & \multicolumn{1}{c|}{$\begin{array}{c}\text { Didaktische } \\
\text { Eigenschaften }\end{array}$} & Glottodidaktische Eigenschaften \\
\hline - ist entschlossen & $\begin{array}{c}\text { - flößt den Schülern Ehrfurcht / Respekt ein } \\
\text { - ist ehrgeizig }\end{array}$ & $\begin{array}{l}\text { - übt im Unterricht alle Sprach- } \\
\text { fertigkeiten }\end{array}$ \\
- ist charismatisch & - flößt seinen Schülern Vertrauen ein & - setzt unterschiedliche fremd- \\
- ist ehrlich & - respektiert seine Schüler & mittel im Unterricht ein (fremd- \\
- ist kultiviert & - behandelt seine Schüler wie Partner / ist & sprachige Filme, YouTube - \\
- ist höflich & nicht hochnäsig & Filme, authentische Texte, mul- \\
- ist genau & - behandelt jeden Schüler gleich / bevorzugt \\
- ist praktisch & keine Schüler & timediale Präsentationen etc.) \\
- ist mutig & - ignoriert die Probleme seiner Schüler nicht & - zeigt den Schülern die Vorteile \\
- schreit nicht & - ist offen für Diskussionen & der Fremdsprachen-beherr- \\
- kann eigene Fehler & - macht sich nicht lustig über seine Schüler & schung. \\
zugeben & - ist ein Vorbild für seine Schüler & \\
\hline
\end{tabular}

Wenn man die in der Tabelle 2 dargestellten Merkmale folgenden Gruppen: Persönlichkeitseigenschaften (gemeint sind Persönlichkeitsmerkmale, darunter solche, die im Schulkontext eine "neue", umfangreichere Bedeutung gewinnen), Wissen, Fähigkeiten sowie Kompetenzen (als Verbindung des Wissens und der Fähigkeit seiner praktischen Anwendung) zuordnet, wird klar, welche Gruppe im Bild eines "guten“ Fremdsprachenlehrers dominiert. In der Tabelle 2 befinden sich insgesamt 105 Merkmale, von denen 60 der Gruppe der Persönlichkeitseigenschaften zugeschrieben wurden. Den zweiten Platz nehmen die wünschenswerten Fähigkeiten eines "guten" Fremdsprachenlehrers mit 29 Beispielen ein, die sich auf die gute Planung und geschickte, systematische Durchführung des Unterrichts direkt beziehen. Die nächste Gruppe mit 9 aufgelisteten Merkmalen bilden die Kompetenzen des Fremdsprachenlehrers, die von den Probanden ganz allgemein (didaktische Kompetenz, glottodidaktische Kompetenz, weit verstandene Sprachkompetenz ${ }^{12}$ ) aufgefasst, oder in Teilkompetenzen (phonetische, grammatische, lexikalische Kompetenz, Medienkompetenz) gegliedert wurden. Die restlichen 7 Merkmale werden der Gruppe Wissen zugewiesen, im Rahmen derer sich weitere Wissensbereiche unterscheiden lassen. Den Probanden zufolge handelte es sich um das umfangreiche Weltwissen und das fachspezifische Wissen, darunter das metasprachliche Wissen und Kulturwissen (gemeint ist das Wissen über die Kultur des Zielsprachenlandes). Die obige Gliederung der Merkmale sowie ihre tabellarische Zusammenstellung zeigen deutlich, dass die Individualität des Lehrers als Menschen für die Ler-

12 Die Kompetenzen, Wissensbereiche, über die ein "guter" Fremdsprachenlehrer verfügen sollte, wurden von den Probanden nicht direkt, mithilfe der Fachterminologie, genannt. Die Studenten zeigten eher die Neigung zur Beschreibung der erwähnten Größen. 
nenden genauso große (oder sogar größere) Rolle spielt wie seine didaktischen Talente und Fähigkeiten. Bei der weiteren Beschreibung des gewonnenen Datenmaterials bleiben wir bei der Einteilung in Persönlichkeits-, didaktische und glottodidaktische Merkmale.

Bei der quantitativen Analyse der erhobenen Daten wurde klar, dass für die Probanden die Persönlichkeitsmerkmale einer Person, von der sie unterrichtet werden, von Belang sind. Geduld, Verständnis, Gerechtigkeit und Kreativität galten in den Augen der befragten Studenten als die Hauptmerkmale, anhand derer entschieden wird, ob man einen (Fremdsprachen)Lehrer als "gut" bezeichnen kann. In ihren zusätzlichen Kommentaren unterstrichen die Studenten folgendes: das Verständnis ist auch eine sehr wichtige Eigenschaft eines Lehrers, denn die Schüler fangen erst ihre Abenteuer mit einer Fremdsprache an und es soll für den Lehrer selbstverständlich sein, dass sie noch Fehler begehen. Außer den oben genannten Charakterzügen, sollten sich den Probanden zufolge großer Anerkennung solche Lehrer erfreuen, die tolerant, ehrlich, selbstbewusst, durchsetzungsfähig, entschlossen, ehrgeizig, charismatisch sind, und dabei Sinn für Humor haben. Die Befragten verwiesen auf weitere Persönlichkeitseigenschaften, die im (Fremdsprachen)Lehrerberuf besonders gefragt sind, wie: Eloquenz, Intelligenz, Kultiviertheit, Höflichkeit, Professionalität und Pünktlichkeit. Ihrer Meinung nach sollte ein "guter" (Fremdsprachen)Lehrer auch locker, dabei aber gut organisiert und praktisch sowie mutig und standhaft sein. Auf gar keinen Fall, so die Studenten, darf der Lehrer im Unterricht schreien - Der Lehrer soll gelassen sein, seine Stimme gegen die Schüler nicht erheben, Ruhe bewahren und anders als mit Geschrei bzw. mit Mahnungen im Klassenbuch für Disziplin im Unterricht sorgen. Ferner sollte er Prinzipien besitzen, an die er sich hält, trotzdem sollte er sich, laut Befragten, nicht zu ernst nehmen. Generell sollte er ein Menschenfreund sein, der gerne mit anderen zusammenarbeitet.

In der Gruppe der didaktischen Merkmale eines "guten" Fremdsprachenlehrers befindet sich an der Spitze die Transparenz des Unterrichtsgeschehens. Der "gute" (Fremdsprachen)Lehrer sollte unbedingt die Fähigkeit der geschickten Wissens- und Stoffvermittlung besitzen. Er sollte den Studenten zufolge das grammatische und lexikalische Wissen klug, gewandt und wohldurchdacht vermitteln. Er sollte den Lernstoff so erklären, dass jedem "was im Kopf bleibt". Manche Studenten notierten noch eine Anmerkung, dass der Lehrer das entstandene sprachliche Problem auf gar keinen Fall ignorieren sollte. Demgemäß sollte er verschiedene Schritte unternehmen, damit die Lernenden Sinn und Funktion der Lerninhalte und Lernaufgaben verstehen. In jeder Unterrichtssituation, in der etwas nicht verstanden wurde, müsste er sich Zeit nehmen und entsprechend reagieren - der Lehrer soll immer Zeit haben, um den unverständlichen Stoff zu erklären. Von den Pro- 
banden wurde nicht nur erwartet, dass ein "guter" (Fremdsprachen)Lehrer den Lernenden Einsichten in Inhalte und Methoden des Unterrichts ermöglicht, sondern auch, dass er Wissensvermittlung mit Leidenschaft verbindet.

Dazu sollte er auf seine Lektionen immer gut vorbereitet sein sowie in den Unterricht zusätzliche didaktische Lernmaterialien einbeziehen, eigene Erfahrungen mit den Schülern teilen und ihnen Informationen vermitteln, die nicht unbedingt in ihren Lehrwerken stehen - Der Lehrer soll Bücher und Lehrwerke als unsere Hilfsmaterialien betrachten, keine Theorien uns daraus vorlesen und verlangen, dass wir uns alles auswendig einprägen; der Lehrer soll kreativ sein, sein Kopf soll voll von unterschiedlichen Ideen sein, er darf nicht ausschließlich anhand des Lehrwerks unterrichten. Die Probanden schätzten Einsatz, Engagement, das lebhafte Interesse des Lehrers für den Unterrichtsinhalt sowie sein Einfallsreichtum, wobei solche Verhaltensweisen man, laut Befragten, nur von gut ausgebildeten und belesenen Lehrern erwarten kann. Der "gute" (Fremdsprachen)Lehrer sollte sich also die Mühe geben, den Unterricht möglichst abwechslungsreich zu gestalten. Um die Motivation seiner Lerner zum unterrichteten Fach aufrechtzuerhalten oder zu steigern, sollte er, je nach dem Thema der Lektion, für einen gezielten Wechsel der Sozialformen sorgen, bedeutungshaltige Übungsformen und Spielübungen (vor allem bei Anfängern) bzw. neueste Technologien (Video, Internet) im Unterricht einsetzen - Sehr wichtig finde ich die pädagogischen Fähigkeiten eines Lehrers, die Arbeit im Unterricht so zu gestalten, dass jeder Schüler an dem Fach Interesse zeigt. Das ermöglichen die abwechslungsreichen Aufgaben, das unterschiedliche Arbeitstempo in jeder Phase des Unterrichts und das Einsetzen der audiovisuellen Materialien. Außer der gut entwickelten Medienkompetenz erwarteten die Probanden von einem "guten" (Fremdsprachen)Lehrer, dass er die Heterogenität der Lernenden respektiert. Im erfolgreichen binnendifferenzierenden (Fremdsprachen)Unterricht sollte der Lehrer jeden einzelnen Schüler individuell behandeln, seine Stärken und Schwächen, sein Arbeitstempo, seine Bedürfnisse sollten im Unterricht berücksichtigt werden - der Lehrer soll mit besonders talentierten Schülern anders arbeiten als mit denen, die Lernschwierigkeiten aufweisen.

Die an der Untersuchung teilnehmenden Studenten hoben hervor, dass ein "guter" (Fremdsprachen)Lehrer den Lernenden gegenüber offen sein sollte. Seine Interaktionsfähigkeiten sind gefragt, er soll sich seinen Schülern gegenüber freundlich verhalten; der Lehrer soll seine Studenten gut behandeln, denn die Lernmotivation steigt, wenn der Lehrer freundschaftliches und respektvolles Verhältnis zu ihnen hat. Resümierend sollte er kein Diktator, sondern ein Freund sein. Er sollte aber nicht nur eine positive Einstellung zu seinen Lernern haben bzw. sie mögen, sondern auch ihnen zuhören und offen auf ihre Ideen und Vorschläge reagieren. 
Das positive Klassenklima hat für die Befragten eine besondere Bedeutung. Der Lehrer sollte für Ruhe und Disziplin sorgen. Die Lerner sollten ihm vertrauen, ihn respektieren, sie sollten aber auch von ihm respektiert und wie Partner des Unterrichtsgeschehens betrachtet werden. Wenn sie Lernschwierigkeiten haben bzw. Probleme, die mit ihrem privaten Leben zusammenhängen, sollte er sie nicht ignorieren, sondern unterstützen - Die Schüler sollen sich dessen bewusst sein, dass der Lehrer so eine Person ist, an die sie sich in jeder Situation und mit jedem Problem wenden können.

Die Untersuchungspersonen widmeten bei dem Antworten auf die gestellte Frage zum Profil eines "guten“ Fremdsprachenlehrers viel Aufmerksamkeit der Bewertung bzw. Benotung ihres Engagements im Unterricht. Ihnen schien es besonders wichtig zu sein, dass alle Schüler nach gleichen Kriterien bewertet werden, keiner von ihnen sollte aus irgendeinem Grunde bevorzugt, keiner wegen seines Scheiterns ausgelacht werden. Dabei sollte das Wissen der Lerner getestet werden, nicht ihre Persönlichkeit. Die Studenten wünschten sich eine regelmäßige Überprüfung ihres Wissens, jedoch unter der Bedingung, dass der Lehrer bei der Benotung konsequent bleibt und das Engagement jedes einzelnen berücksichtigt sowie dass er im Falle einer negativen Note den Lernenden eine Chance gibt, die zu verbessern.

Die Probanden wollten in ihrem Lehrer ein Vorbild sehen, d. h. einen Menschen, dem der ausgeübte Beruf keine Dienstleistung den anderen gegenüber bedeutet, sondern seine größte Leidenschaft ist - Die Passion macht aus einem Lehrer einen Künstler, einen Meister in seinem Fach (dazu braucht man selbstverständlich bestimmtes Wissen), der im Gegensatz zu einfachen Handwerkern, nicht mit einem harten und stumpfen Meißel ein bisschen Wissen aus seinen Lernern zu gewinnen versucht. Diese Aussage wurde mit der Feststellung ergänzt, dass einen guten Fremdsprachenlehrer nicht nur die Begeisterung für das Unterrichten auszeichnen soll, sondern für die Sprache (ihren Klang, ihre Struktur), aber auch für die Kultur, Geschichte und Tradition des Landes, aus dem diese Sprache stammt. Diese Äußerung korrespondiert direkt mit der nächsten Gruppe von Merkmalen und zwar mit den glottodidaktischen Merkmalen, die sich schon explizit auf den Fremdsprachenunterricht beziehen.

Die von den Probanden angegebenen glottodidaktischen Eigenschaften lassen sich zwei Gruppen von Kompetenzen zuordnen, in die jeder "gute" Fremdsprachenlehrer ausgestattet werden sollte. Es handelt sich um Fachkompetenz (darunter Sprach- und Kulturkompetenz sowie kommunikative und metasprachliche Kompetenz) und didaktische Kompetenz. Die Studenten wünschten sich vor allem einen kompetenten Fremdsprachenlehrer, der perfekt bzw. gut die Sprache beherrscht, die er unterrichtet. Er sollte in dieser Sprache fließend kommunizieren können, am besten ohne Akzent. Eine gute Aussprache ist aber nicht ausreichend, um ein Experte in seinem Fach 
zu werden, dazu zählten, laut Befragten, noch ein reiches Vokabular, gute Grammatikaneignung, das umfangreiche Wissen über die Sprache selbst sowie Kenntnis der kulturellen Gegebenheiten bezüglich der Geschichte, Literatur, Kunst usw. - Ein guter Fremdsprachenlehrer soll vor allem die unterrichtete Sprache richtig können und nicht nur das Diplom besitzen, das seinen Schulabschluss bestätigt. Und dabei ist es irrelevant, in welcher Schule (Grundschule, Oberschule) er unterrichten wird; Er soll das Allgemeinwissen besitzen, um mit Studenten über verschiedene Themen diskutieren zu können.

All die gerade genannten Kompetenzen (phonetische, lexikalische, grammatische Kompetenz sowie Kulturkompetenz) müssen noch um eine Gruppe der Fähigkeiten ergänzt werden, die sich direkt mit dem Verlauf des Fremdsprachenunterrichts verbinden. Die Untersuchungspersonen legten einen großen Wert auf die geschickte Planung, systematische Durchführung und Kontrolle des schulischen Lernens, vor allem auf die Vermittlungskünste der Lehrperson im grammatischen und lexikalischen Bereich. Es wurde von ihnen hervorgehoben, dass ein "guter" Fremdsprachenlehrer nicht nur ein guter Sprachvermittler sein sollte, sondern ein Wegweiser, der ihnen die effektivsten Methoden und Strategien der Sprachbeherrschung zeigt. Bei dieser Tätigkeit sollte er unterschiedliche Lehrmittel bzw. Technologien benutzen, die die jungen, modernen Menschen begeistern können (YouTube, Internet, Smartphone, multimediale Präsentationen u. a.) - Internet ist die Zukunft des Unterrichtens, ich will von einem modernen Lehrer unterrichtet werden, der sich mit der neuen Technik und neuesten Möglichkeiten im Bereich der Fremdsprachenvermittlung und-aneignung bestens auskennt.

Darüber hinaus sollte der Fremdsprachenlehrer das Arbeitstempo und Aufgaben dem Sprachniveau der Studenten anpassen, was schon teilweise bei der Präsentation der didaktischen Eigenschaften eines "guten“ Lehrers thematisiert wurde. Ferner sollte er über die Fähigkeit verfügen, die Studenten zur fremdsprachlichen Kommunikation, $\mathrm{zu}$ freiem und kreativem Sprachverhalten zu motivieren, indem er erstens selbst im Unterricht ausschließlich die Fremdsprache benutzt und zweitens den Studenten die Vorteile der praktischen Fremdspracheanwendung zeigt. Sehr vorsichtig und taktvoll sollte er aber an die Fehlerkorrektur herangehen. Die Probanden plädierten für eine positive Korrektur, bei der sie keine Angst haben müssen, vor der ganzen Gruppe ausgelacht oder blamiert zu werden. Sie träumten von einem Lehrer, der sie indirekt, $d$. h. erst nach der abgeschlossenen Äußerung verbessert und die Selbstkorrektur sowohl im schriftlichen als auch im mündlichen Bereich zulässt - Der Lehrer soll uns zum Sprechen motivieren, unsere Fehler erst dann korrigieren, wenn wir mit unserer Aussage fertig sind. Sonst lenkt er uns nur ab und verwirrt uns. Bei den Studenten verschafften sich solche Lehrer besondere Achtung, die auch ihre eigenen Fehler zugeben 
können - Er soll nicht so tun, als ob er alles gewusst hätte z. B. wenn er ein Wort vergessen hat, soll er das zugeben; der Lehrer soll das gestehen, wenn er etwas nicht weiß bzw. wenn er etwas vergessen hat, es gibt nämlich keine Menschen, die alles wissen.

Resümierend kann man sagen, dass der Fremdsprachenlehrer alles, was in seiner Macht steht, tun sollte, um den Erwerbsprozess einer nicht immer einfachen Fremdsprache so angenehm, wie möglich, zu gestalten. Dabei spielen seine Persönlichkeitsmerkmale und sein Auftreten den Lernenden gegenüber eine entscheidende Rolle. Die Probanden verwiesen in ihrer Analyse auch auf Schwerpunkte, die sowohl mit didaktischen Fähigkeiten einer Lehrperson als auch mit ihren Talenten zur Organisierung des Lernprozesses in Korrelation stehen.

\section{ABSCHLIESSENDE BEMERKUNGEN}

Die Gruppe der Hochschullerner, die an der gerade präsentierten Untersuchung beteiligt war, war gleichzeitig die letzte Gruppe, die von uns untersucht wurde. So gingen wir an die Analyse der erhobenen Daten mit der Erfahrung heran, dass die Persönlichkeitseigenschaften eines Lehrers in der Rangordnung ihrer Wichtigkeit über seine (glotto)didaktischen Merkmale dominieren. Jedoch erhofften wir uns von den zukünftigen Lehrkräften, dass sie mehr Aufmerksamkeit der fachwissenschaftlichen Vorbereitung des Fremdsprachenlehrers auf seine schulische Tätigkeit schenken werden. Teilweise wurden unsere Erwartungen erfüllt, denn Neuphilologie-Studierende befassten sich bei der Antwort auf die gestellte Frage zum Profil eines "guten“ Fremdsprachenlehrers sowohl mit seinen pädagogischen Kompetenzen als auch mit dem von ihm geleiteten Verlauf einer Fremdsprachenstunde, wobei man hervorheben muss, dass lediglich eins von all den aufgezählten didaktischen Merkmalen von mehr als ein Viertel der Befragten angesprochen wurde. Trotz der von den Untersuchungspersonen genannten Eigenschaften bezüglich der Vermittlung des Lernstoffes, der Vielfalt der im Unterricht eingesetzten Methoden, Lernmaterialien etc. sowie der direkten Arbeit des Lehrers mit seinen Schülern (Individualisierung, Motivation, Fehlerkorrektur, Bewertung usw.) zählten für die werdenden Lehrer in erster Linie die Eigenschaften, die direkt mit dem Charakter einer Lehrkraft zusammenhängen. Absolute Spitzenreiter waren Geduld und Verständnis, die fast für die Hälfte der Probanden einen "guten" Fremdsprachenlehrer auszeichnen sollten, über ein Drittel der Befragten hielt Gerechtigkeit und Kreativität im Unterrichtsgeschehen für wünschenswert. Diese Ergebnisse zeigen deutlich, dass die (glotto)didaktischen Fähigkeiten und Kompetenzen eines 
Lehrers im institutionellen Fremdsprachenlernen eher eine sekundäre Rolle spielen. Einerseits sind diese Schlussfolgerungen erschreckend, weil, wie schon im ersten Punkt dieses Beitrags erwähnt, bilden die Persönlichkeitsmerkmale einer zukünftigen Lehrkraft (auch wenn sie für die untersuchte Gruppe von größter Bedeutung sind) kein Qualifikationskriterium für den Lehrerberuf. Außerdem finden sie keinen festen Platz bei den für die Lehrerausbildung vorgesehener Standards. Andererseits ist dieser Sachverhalt legitim, denn heutzutage, in der Zeit, in der in jeder neuphilologischen Konferenz, in jedem Seminar über die Lehrerausbildung bzw. Lehrerrolle etc. die Rede ist, scheint die Frage nach dem Profil eines "guten“ Lehrers in Bezug auf seine Persönlichkeitseigenschaften für viele Wissenschaftler oberflächlich und uninteressant zu sein. Es ist uns völlig klar, dass viele von den genannten Merkmalen als subjektive Werte betrachtet werden, die eigentlich gar nicht messbar sind, wir möchten jedoch darauf hinweisen, dass es bei der Persönlichkeit eines Lehrers um viel mehr geht als nur um nett bzw. humorvoll zu sein. Unserer Meinung nach, muss man schon viel tiefer das Wesen einer Person unter die Lupe nehmen, um ihren Charakter für den Lehrerberuf als geeignet bzw. nicht geeignet zu klassifizieren. Das ideale Bild, das mit Sicherheit in seiner reinsten Form nicht existiert, wird durch die Verflechtung von all den erwähnten Merkmalen entstehen. Die werdenden Lehrer können aber anhand der durchgeführten Analyse erfahren, welches Ziel sie in ihrem zukünftigen Berufsleben verfolgen sollen.

Die von uns beschriebene Untersuchung und ihre Ergebnisse berücksichtigen zwar ausschließlich den polnischen Bildungskontext, beziehen sich jedoch, unserer Meinung nach, wegen der teilweise gemeinsamen Bildungspolitik der EU-Länder auf andere europäische Bildungskontexte und können deswegen als ein Wegweiser für diejenigen gelten, die die grundlegenden Entscheidungen über die Form der Fremdsprachenlehrerausbildung in der europäischen Gemeinschaft treffen.

\section{LITERATURVERZEICHNIS}

Andrzejewska, E. (Hrsg.) (2015a). Kształcenie nauczycieli języków obcych. Neofilolog, 44 (2).

Andrzejewska, E. (Hrsg.) (2015b). Nauczyciele języków obcych wobec zadań współczesnej glottodydaktyki. Neofilolog, 45 (2).

Aleksandrowicz-Pędich, L. / Komorowska, H. (Hrsg.) (1999). Ksztatcenie nauczycieli języków obcych w Polsce: narodziny systemu - przykłady funkcjonowania - potrzeby. Białystok: Wydawnictwo Uniwersytetu w Białymstoku.

Ausubel, D.-P. / Novak, J.-D. / Hanesian, H. (1980). Psychologie des Unterrichts. Bd. 2. Weinheim, Basel: Beltz.

Baley, S. (1958/1959). Psychiczne właściwości nauczyciela-wychowawcy. In: W. Okoń (Hrsg.), Osobowość nauczyciela (S. 170-209). Warszawa: Państwowe Zakłady Wydawnictw Szkolnych. 
Bandura, L. (1972). O procesie uczenia się. Warszawa: Państwowe Zakłady Wydawnictw Szkolnych.

Dawid, J.-W. (1912/1959). O duszy nauczycielstwa. In: W. Okoń (Hrsg.), Osobowość nauczyciela (S. 33-51). Warszawa: Państwowe Zakłady Wydawnictw Szkolnych.

Diekmann, A. (2007). Empirische Sozialforschung. Grundlagen, Methoden, Anwendungen. Hamburg: Rowohlt.

Düwel, H. (1983). Motivierung im Fremdsprachenunterricht in Abhängigkeit einzelner Unterrichtsfaktoren. In: G. Solmecke (Hrsg.), Motivation und Motivieren im Fremdsprachenunterricht (S. 179-198). Paderborn u. a.: Schöningh.

Dzierzbicka, W. (1926). O uzdolnieniach zawodowych nauczyciela-wychowawcy. Lwów-Warszawa: Książnica-Atlas.

Grucza, F. (Hrsg.) (1988). Problemy kształcenia nauczycieli języków obcych. Materiały z XI Ogólnopolskiego Sympozjum zorganizowanego przez Instytut Lingwistyki Stosowanej UW w Jaszowcu, 7-10 listopada 1985. Warszawa: Wydawnictwa Uniwersytetu Warszawskiego.

Grucza, F. / Krumm, H.-J. / Grucza, B. (Hrsg.) (1993). Beiträge zur wissenschaftlichen Fundierung der Ausbildung von Fremdsprachenlehrern. Warszawa: Wydawnictwa Uniwersytetu Warszawskiego.

Grzegorzewska, M. (1947/2002). Listy do Młodego Nauczyciela. Cykl I-III. Warszawa: Wydawnictwo Akademii Pedagogiki Specjalnej.

Hattie, J. (2014). Lernen sichtbar machen. Überarbeitete deutschsprachige Ausgabe von "Visible Learning" von Wolfgang Beywl und Klaus Zierer. Hohengehren: Schneider.

Hattie, J. / Zierer, K. (2018a). Kenne deinen Einfluss!: „Visible Learning“ für die Unterrichtspraxis. Hohengehren: Schneider.

Hattie, J. / Zierer, K. (2018b). Visible Learning: Auf den Punkt gebracht. Hohengehren: Schneider.

Kindler, W. (2016). Die Lehrerpersönlichkeit - Chance und Herausforderung: Wie Sie Ihre Rolle finden und Lernerfolg steigern. Mühlheim an der Ruhr: Verlag an der Ruhr.

Komorowska, H. (1982). Metody badań empirycznych w glottodydaktyce. Warszawa: PWN.

Kreutz, M. (1947/1959). Osobowość nauczyciela-wychowawcy. In: W. Okoń (Hrsg.), Osobowość nauczyciela (S. 113-164). Warszawa: Państwowe Zakłady Wydawnictw Szkolnych.

Krzysiak, L. (2013). Neue Standards für die Lehrerbildung in Polen: Merkmale, Funktionen und mögliche Folgen. In: L. Krzysiak (Hrsg.), Blickpunkte der Germanistik. Literatur- und Kulturwissenschaft, Linguistik und Fremdsprachendidaktik (S. 155-164). Frankfurt am Main: Peter Lang $\mathrm{GmbH}$.

Krzysiak, L. (2016). Ausbildung von Fremdsprachenlehrenden: Standards für die Lehrerbildung im europäischen Kontext. In: K. Mihułka / M. Sieradzka / R. Budziak (Hrsg.), Die Fremdsprache Deutsch in Polen: Anfänge, Gegenwart, Perspektiven (S. 133-145). Rzeszów: Wydawnictwo Uniwersytetu Rzeszowskiego.

Kuen, G. / Schwarzmann, A. (2013). So geht guter Unterricht. Schüler geben Referendaren Tipps. Weinheim: Beltz.

Loebell, P. / Martzog, P. (Hrsg.) (2016). Wege zur Lehrerpersönlichkeit: Kompetenzerwerb, Persönlichkeitsentwicklung und aktuelle Herausforderungen in der Lehrerbildung. Leverkusen: Barbara Budrich Verlag.

Łobocki, M. (2009). Wprowadzenie do metodologii badań pedagogicznych. Kraków: Oficyna Wydawnicza Impuls.

Mihułka, K. (2018a). Osobowość nauczyciela a wpływ wychowawczy - ujęcie teoretyczne. Jezzyki Obce w Szkole, 3, 119-125.

Mihułka, K. (2018b). Walory osobowe nauczyciela w opinii uczniów - przegląd współczesnych badań. Jezzyki Obce w Szkole, 4, 99-103. 
Mihułka, K. / Chojnacka-Gärtner, J. (2017a). Forschungsbericht zum Profil eines "guten" Fremdsprachenlehrers am Gymnasium. Linguodidactica, XXI, 205-219.

Mihułka, K. / Chojnacka-Gärtner, J. (2017b). Forschungsbericht zum Profil eines "guten" Fremdsprachenlehrers in der Grundschule. Studia Niemcoznawcze, 60, 977-992.

Mihułka, K. / Chojnacka-Gärtner, J. (2018). Forschungsbericht zum Profil eines "guten“ Fremdsprachenlehrers in der Oberschule. Orbis Linguarum, 51, 647-660.

Mihułka, K. / Chojnacka-Gärtner, J. (2019). „Auf die Persönlichkeit des Lehrers kommt alles an [...]" - Forschungsbericht zum Profil eines "guten“ Fremdsprachenlehrers in allen Schultypen in Polen, Journal of Languages for Specific Purposes, 6, 167-176.

MNiSW = Minister Nauki i Szkolnictwa Wyższego. (2012). Rozporządzenie Ministra Nauki i Szkolnictwa Wyższego z dnia 17 stycznia 2012 r. w sprawie standardów kształcenia przygotowujących do wykonywania zawodu nauczyciela. Dziennik Ustaw, poz. 131, http:/ / dziennikustaw.gov.pl/du/2012/131/1 (Zugriff am: 22.07.2018).

Mysłakowski, Z. (1925/1959). Co to jest "talent pedagogiczny"? In: W. Okoń (Hrsg.), Osobowość nauczyciela (S. 57-68). Warszawa: Państwowe Zakłady Wydawnictw Szkolnych.

Okoń, W. (1959). Problem osobowości nauczyciela. In: W. Okoń (Hrsg.), Osobowość nauczyciela (S. 2-28). Warszawa: Państwowe Zakłady Wydawnictw Szkolnych.

Pawlak, M. / Mystkowska-Wiertelak, A. / Pietrzykowska, A. (Hrsg.) (2009). Nauczyciel języków obcych dziś i jutro. Poznań-Kalisz: Wydział Pedagogiczno-Artystyczny UAM w KaliszuUAM w Poznaniu.

Pfeiffer, W. (2001). Nauka języków obcych. Od praktyki do praktyki. Poznań: Wagros.

Stańczyk, J. (Hrsg.) (2013). Kompetencje ogólne. Kształcenie nauczycieli. Neofilolog, 40 (2).

Szuman, S. (1947/1959). Talent pedagogiczny. In: W. Okoń (Hrsg.), Osobowość nauczyciela (S. 73-108). Warszawa: Państwowe Zakłady Wydawnictw Szkolnych.

Wawrzyniak-Śliwska, M. (Hrsg.) (2015). Kształcenie i doskonalenie nauczycieli języków obcych - potencjał i potrzeby. Neofilolog, 45 (1).

Wilczyńska, W. / Michońska-Stadnik, A. (2010). Metodologia badań w glottodydaktyce. Wprowadzenie. Kraków: AVALON.

Wysocka, M. (2003). Profesjonalizm w nauczaniu języków obcych. Katowice: Wydawnictwo Uniwersytetu Śląskiego.

Zawadzka, E. (2004). Nauczyciele języków obcych w dobie przemian. Kraków: Impuls.

Received: 21.08.2018; revised: 18.03.2019 